\title{
LIBROS RECIBIDOS
}

Abellán, José Luis. La idea de América: origen y evolución. Madrid: Iberoamericana, 2009.

Adorno, Rolena. De Guancane a Macondo: Estudios de Literatura Hispanoamericana. Sevilla: Editorial Renacimiento, 2008.

Aldaz, Anna-Marie (ed.). An Anthology of Nineteenth-Century Women's Poetry from Spain: In English translation, with Original Text. New York: MLA, 2009.

Alemany Bay, Carmen, y Beatriz Aracil Varón (eds.) América en el imaginario europeo: Estudios sobre la idea de América a lo largo de cinco siglos. Alicante: Publicaciones de la Universidad de Alicante, 2009.

Alonso, Diego. José Enrique Rodó: una retórica para la democracia. Montevideo: Ediciones Trilce, 2009.

Altuna, Elena. Retórica del desagravio: estudios de cultura colonial peruana. Salta: CEPIHA, Universidad Nacional de Salta, 2009.

Alvarez Borland, Isabel y Lynette M. F. Bosch. (eds.) Cuban-American Literature and Art: Negotiating Identities. Albany: State U of New York P, 2009.

Arellano, Ignacio y Jesús M. Usunáriz (eds.) El mundo social y cultural de la celestina. Madrid: Iberoamericana, 2009.

Arguedas, José María. Qepa Winaq... siempre literatura y antropología. (prólogo de Sybila Arguedas, edición crítica de Sora Sales). Madrid: Iberoamericana, 2009.

Aszyk, Urszula (coord.). El espacio dramático frente al espacio escénico. Varsovia: Instituto de Estudios Ibéricos e Iberoamericanos de la Universidad de Varsovia, 2003.

Baeza Ventura, Gabriela y Marc Zimmerman (coords.) Estudios culturales centroamericanos en el nuevo milenio. San José: Editorial UCR, 2009.

Barrientos, Juan José. La gata revolcada. Veracruz: Instituto Veracruzano de la Cultura, 2009. 
Bauer, Ralph y José Antonio Mazzotti (eds.). Creole Subjects in the Colonial Americas: Empires, Texts, Identities. Chapel Hill: U of North Carolina P, 2009.

Berg, Mary G., Pamela Carmell y Anne Fountain (eds.). Cuba on the Edge: Short Stories From the Island. Nottingham: CCC Press, 2007.

Bergmann, Emilie L. y Stacey Schlau (eds.). Approaches to Teaching the Works of Sor Juana Inés de la Cruz. New York: MLA, 2007.

Bermúdez Barrios, Nayibe. Sujetos transnacionales: la negociación en cine y literatura. Ciudad Juárez: Universidad Autónoma de Ciudad Juárez, 2008.

Biron, Rebecca E. (ed.). City/Art. Durham: Duke UP, 2009.

Blake, Charles H. y Stephen D. Morris (eds.). Corruption \& Democracy in Latin America. Pittsburgh: U of Pittsburgh P, 2009.

Braig, Marianne y Anne Huffschmid (eds.). Los poderes de lo público: Debate, espacios y actores en América Latina. Madrid: Iberoamericana, 2009.

Capetillo, Luisa (translation and introduction by Lara Walker.). Absolute Equality: An Early Feminist Perspective, Influencias de las ideas modernas. Houston: Arte Público Press, 2009.

Castillo, Moisés R. Indios en la escena: la respresentación del amerindio en el teatro del Siglo de Oro. Lafayette: Purdue UP, 2009.

Cejudo, Francisco. Tierras prometidas. Córdoba: Editorial Ánfora Nova, 2009.

Celorio, Gonzalo. And Let the Earth Tremble at its Centers. Dick Gerdes, trad. Notas por Rubén Gallo. Austin: U of Texas P, 2009.

Cervantes, Miguel. Don Quixote. James H. Montgomery, trad. y notas. Introducción por David Quint. Indianapolis: Hackett Publishing Company, 2009.

Colom González, Francisco (ed.). Modernidad iberoamericana: cultura, política y cambio social. Madrid: Iberoamericana, 2009.

Copertari, Gabriela. Desintegración y justicia en el cine argentino contemporáneo. Woodbridge: Tamesis, 2009.

Coronado, Jorge. The Andes Imagined: Indigenismo, Society, and Modernity. Pittsburgh: U of Pittsburgh P, 2009

Cruz, Sor Juana Inés de la. Neptuno alegórico. Vincent Martin y Electa Arenal, eds. Madrid: Ediciones Cátedra, 2009.

Del Valle, Ivonne. Escribiendo desde los márgenes: colonialismo y jesuitas en el siglo XVII. México D.F.: Siglo Veintiuno Editores, 2009.

D’Escoto, Miguel. Antiimperialismo y noviolencia. Mexico D.F.: Ocean Sur, 2009.

Di John, Jonathan. From Windfall to Curse? Oil and Industrialization in Venezuela, 1920 to the Present. University Park: Pennsylvania State UP, 2009. 
Eichmann Oehrli,Andrés. Cancionero mariano de Charcas. Madrid: Iberoamericana, 2009.

Escobar, Anna María y Wolfgang Wölck (eds.). Contacto lingüístico y la emergencia de variantes y variedades lingüísticas. Madrid: Iberoamericana, 2009.

Estrada, Oswaldo. La imaginación novelesca: Bernal Díaz entre géneros y épocas. Madrid: Iberoamericana, 2009.

Fedewa, Marilyn H. María of Ágreda: Mystical Lady in Blue. Albuquerque: U of New Mexico P, 2009.

Fitz, Earl E. Brazilian Narrative Traditions in a Comparative Context. New York: MLA, 2005.

Frohlich, Margaret G. Framing the Margin: Nationality and Sexuality Across Borders. Tempe: AILCFH, 2008.

Garrels, Elizabeth (ed.). Ifigenia: Diario de una señorita que escribió porque se fastidiaba, por Teresa de la Parra. Doral: Stockcero, 2008.

Gómez, Leila. Iluminados y tránsfugas: relatos de viajeros y ficciones nacionales en Argentina, Paraguay y Perú. Madrid: Iberoamericana, 2009.

González Álvarez, José Manuel. En los 'bordes fluidos': Formas híbridas y autoficción en la escritura de Ricardo Piglia. Bern: Peter Lang AG, 2009.

González Boixo, José Carlos. Tendencias de la narrativa mexicana actual. Madrid: Iberoamericana, 2009.

Gordon, Richard A. Cannibalizing the Colony: Cinematic Adaptations of Colonial Literature in Mexico and Brazil. West Lafayette: Purdue UP, 2009.

Grützmacher, Łucasz. ¿El descubridor descubierto o inventado? Cristóbal Colón como protagonista en la novela histórica hispanoamericana y española de los últimos 25 años del siglo XX. Varsovia: Bilioteka Iberyjska, 2009.

Gustafson, Bret. New Languages of the State: Indigenous Resurgence and the Politics of Knowledge in Bolivia. Durham: Duke UP, 2009.

Hardouin-Fugier, Elisabeth. Bullfighting: A Troubled History. London: Reaktion Books, 2009.

Indych-López, Anna. Muralism Without Walls: Rivera, Orozco, and Siqueiros in the United States, 1927-1940. Pittsburgh: U of Pittsburgh P, 2009.

Keenaghan, Eric. Queering Cold War Poetry: Ethics of Vulnerability in Cuba and the U.S. Columbus: Ohio State UP, 2009.

Kulawik, Krzysztof. Travestismo lingüístico: El enmascaramiento de la identidad sexual en la narrativa latinoamericana neobarroca. Madrid: Iberoamericana, 2009.

Kumor, Karolina. De Cervantes a Calderón: estudios sobre la literatura y el teatro español del Siglo de Oro, una homenaje al Profesor Kazimierz Sabik. Varsovia: Instituto de Estudios Ibéricos y Iberoamericanos, 2009. 
La Fountain-Stokes, Lawrence. Queer Ricans: Cultures and Sexualities in the Diaspora. Minneapolis: University of Minnesota Press, 2009.

La Fountain-Stokes, Lawrence. Uñas pintadas de azul/Blue Fingernails. Tempe: Bilingual Press, 2009.

Linde-Usiekniewicz, Jadwiga y Ana Enriquez-Vicentefranquiera (coor.) Estudios en lingüística ibérica e iberoamericana. Varsovia: Instituto de Estudios Ibéricos e Iberoamericanos de la Universidad de Varsovia, 2006.

Llanos, Bernardita M. Passionate Subjects/Split Subjects in Twentieth-Century Literature in Chile. Lewisburg: Bucknell UP, 2009.

López García, Ángel. La lengua común en la España plurilingüe. Madrid: Iberoamericana, 2009.

Maglia, Graciela. De la machina imperial a la vereda tropical: poesía, identidad y nación en el Caribe afrohispánico. Bogotá: Editorial Pontificia Universidad Javeriana, 2009.

Marzec, Zofia. El opispo Pedro Casaldáliga: poeta de liberación, defensor del otro. El contexto de su poesía y pensamiento. Varsovia: Instituto de Estudios Ibéricos e Iberoamericanos de la Universidad de Varsovia, 2006.

McGuirk, Bernard. Falklands-Malvinas: An Unfinished Business. Seattle: New Ventures, 2007.

McGuirk, Bernard y Else R. P. Vieira (eds.). Haroldo de Campos in Conversation. London: Zoilus Press, 2009.

Millás, Juan José. Personality Disorders and Other Stories. Gregory P. Kaplan, trad. New York: MLA, 2007.

Morel, Hortensia. Palabras en las tablas: ensayos de literatura latinoamericana contemporánea. Río Piedras: La Editorial Universidad de Puerto Rico, 2008.

Moszczyńska, Katarzyna. La vida devorada (novela, mujer y sociedad en la España de los noventa). Varsovia: Instituto de Estudios Ibéricos e Iberoamericanos de la Universidad de Varsovia, 2009.

Muñoz, Sonia. Los devaneos del docto: El caso de la teoría del consumo cultural en América Latina. Cali: Archivos del Índice, 2009.

Nahson, Daniel. La crítica del mito: Borges y la literatura como sueño de vida. Madrid: Iberoamericana, 2009.

Nalewajko, Małgorzata. Identidades: etnias, culturas, naciones. Varsovia: Instituto de Estudios Ibéricos e Iberoamericanos de la Universidad de Varsovia, 2004.

Page, Joanna. Crisis and Capitalism in Contemporary Argentine Cinema. Durham: Duke UP, 2009.

Pancrazio, James J. Enriqueta. Faber: travestismo, documentos e historia. Madrid: Editorial Verbum, 2008. 
Perrone, Charles A. Brazil, Lyric, and the Americas. Gainesville: UP of Florida, 2010.

Pescador, Juan Javier. Crossing Borders with the Santo Niño de Atocha. Alburquerque: U of New Mexico P, 2009.

Pieper Mooney, Jadwiga E. The Politics of Motherhood: Maternity and Women's Rights in Twentieth-Century Chile. Pittsburgh: U of Pittsburgh P, 2009.

Poma de Ayala, Felipe Guaman. The First New Chronicle and Good Government: On the History of the World and the Incas up to 1615 (trans. and ed. Roland Hamilton). Austin: U of Texas P, 2009.

Portela, M. Edurne. Displaced Memories: The Poetics of Trauma in Argentine Women's Writing. Lewisburg: Bucknell UP, 2009.

Poyo, Gerald E. y Tomás Ybarra-Frausto (eds.). Recovering the U.S. Hispanic Literary Heritage,Volume VII. Houston: Arte Público Press, 2009.

Quintana Millamoto, María Esther. Crónicas del Bufón: Aproximación crítica a Maluco, la novela de los descubridores. Montevideo: Tradinco, 2009.

Remenyik, Zsigmond. El lamparero alucinado. László Scholz, ed. Madrid: Iberoamericana, 2009.

Reyes, Alfonso. 20 epistolarios rioplatenses. Serge I. Zaïtzeff, comp. México D.F.: El Colegio Nacional, 2008.

Reyes, Alfonso. Más epistolarios rioplatenses. Serge I. Zaïtzeff, ed. México D.F.: Universidad Autónoma Metropolitana, 2009.

Rodríguez, Ana Patricia. Dividing the Isthmus: Central American Transnational Histories, Literatures and Cultures. Austin: U of Texas P, 2009.

Rodríguez, Ileana. Liberalism at its Limits. Pittsburgh: U of Pittsburgh P, 2009.

Rojo, Grínor. Borgeana. Santiago: Lom Ediciones, 2009.

Royo, Amelia. Imposturas del ensayo. Córdoba: Ediciones Recovecos, 2009.

Sadlier, Darlene J. Latin American Melodrama. Urbana: U of Illinois P, 2009.

Saraceni, Gina. Escribir hacia atrás: herencia, lengua, memoria. Rosario: Beatriz Viterbo Editora, 2008.

Schiwy, Freya. Indianizing Film: Decolonization, the Andes, \& the Question of Technology. New Brunswick: Rutgers UP, 2009.

Scott, Renée S. What is Eating Latin American Women Writers? Food, Weight, and Eating Disorders. Amherst: Cambria Press, 2009.

Simón, Gabriela (comp.). Crónicas Argentinas: La década del 90: literatura y medios. Córdoba: Alción Editora, 2007.

Sklodowska, Elzbieta. Espectros y espejismos: Haití en el imaginario cubano. Madrid: Iberoamericana, 2009.

Sosiński, Marcin(ed.). Trabajos lingüísticos y literarios: cinco años de colaboración entre el Departamento de Lengua Española de la Universidad de Granada 
y el Instituto de Estudios Ibéricos e Iberoamericanos de la Universidad de Varsovia. Varsovia: Universidad de Varsovia, 2009.

Sotomayor Tribín, Hugo A. y Zoilo Cuéllar-Montoya (coords.). Aproximaciones a la paleopatología en América Latina. Bogotá: Convenio Andrés Bello, 2007.

Smith, Matthew J. Red \& Black in Haiti: Radicalism, Conflict, and Political Change 1934-1957. Chapel Hill: U of North Carolina P, 2009.

Vicuña, Cecilia y Ernesto Livon-Grosman (eds.) The Oxford Book of Latin American Poetry. Oxford: Oxford UP, 2009.

Voigt, Lisa. Writing Capitivity in the Early Modern Atlantic. Chapel Hill: U of North Carolina P, 2008.

Waluch-de la Torre, Edyta. Diccionario teórico-práctico de preposiciones espaciales: aplicación traductológica del español al portugués y al polaco. Varsovia: Instituto de Estudios Ibéricos e Iberoamericanos de la Universidad de Varsovia, 2009.

Waluch-de la Torre, Edyta. Preposiciones espaciales en español, portugués y polaco: teoría y evolución. Varsovia: Instituto de Estudios Ibéricos e Iberoamericanos de la Universidad de Varsovia, 2008.

Weber, Alison (ed.) Approaches to Teaching: Teresa of Ávila and the Spanish Mystics. New York: MLA, 2009.

Weiss, Mark (ed.). The Whole Island: Six Decades of Cuban Poetry, A Bilingual Anthology. Berkeley: University of California Press, 2009.

Weldt-Basson, Helene Carol. Subversive Silence: Nonverbal Expression and Implicit Narrative Strategies in the Works of Latin American Women Writers. Madison: Fairleigh Dickinson University Press, 2009.

Zevallos Aguilar, Ulises Juan. Las provincias contraatacan: regionalismo y anticentralismo en la literatura peruana del siglo XX. Lima: Universidad Nacional Mayor de San Marcos, 2009.

Zivin, Erin Graff. The Wandering Signifier: Rhetoric of Jewishness in the Latin American Imaginary. Durham: Duke University Press, 2008.

Zoungbo, Victorien Lavou, and Marlène Marty (eds.). Imaginaire racial et projections identitaires. Perpignan: Presses Universitaires de Perpignan, 2008. 